\title{
Clinical relevance of the relationship between changes in gut microbiota and bile acid metabolism in patients with intrahepatic cholangiocarcinoma
}

\author{
Elisa Herraez $^{1,2}$, Marta R. Romero ${ }^{1,2}$, Rocio I. R. Macias $^{1,2}$, Maria J. Monte ${ }^{1,2}$, Jose J. G. Marin ${ }^{1,2}$ \\ ${ }^{1}$ Experimental Hepatology and Drug Targeting (HEVEFARM), IBSAL, University of Salamanca, Salamanca, Spain; ${ }^{2}$ National Institute for the Study \\ of Liver and Gastrointestinal Diseases (CIBERehd), Carlos III National Health Institute, Madrid, Spain \\ Correspondence to: Jose J. G. Marin. Experimental Hepatology and Drug Targeting (HEVEFARM), IBSAL, University of Salamanca, Campus Miguel \\ de Unamuno E.I.D. S-09, 37007 Salamanca, Spain. Email: jjgmarin@usal.es. \\ Provenance and Peer Review: This article was commissioned by the Editorial Office, Hepatobiliary Surgery and Nutrition. The article did not undergo \\ external peer review. \\ Comment on: Jia X, Lu S, Zeng Z, et al. Characterization of gut microbiota, bile acid metabolism, and cytokines in intrahepatic cholangiocarcinoma. \\ Hepatology 2019. [Epub ahead of print].
}

Submitted Oct 08, 2019. Accepted for publication Oct 15, 2019.

doi: $10.21037 /$ hbsn.2019.10.11

View this article at: http://dx.doi.org/10.21037/hbsn.2019.10.11

Although only $10-15 \%$ of liver cancers are cholangiocarcinomas (CCAs), which derive from the epithelial cells of the biliary tree, these cancers constitute a serious and growing health problem worldwide. The rate of mortality due to this tumor is very high because CCA usually appears in aged people and very often, in more than $70 \%$ of cases, are diagnosed late because of: (I) their anatomical localization in a big organ like the liver; (II) the lack of specific symptoms during most time of their development; and (III) the absence of available accurate, specific and sensitive biomarkers (1). These characteristics reduce the rate of success of surgical resection, which is the only curative option for CCA patients, because the response of this aggressive cancer to the available pharmacological armamentarium is extremely feeble (2). This justifies the interest of many groups to identify non-invasive markers to achieve early diagnosis that also permit to distinguish CCA from non-malignant biliary diseases and from hepatocellular carcinoma (HCC), the most frequent liver cancer, which derives from hepatocytes (3).

In recent years, the intestinal microbiota has been the focus of interest for many studies in the fields of gastroenterology and hepatology because of its role in the development and progression of liver and gastrointestinal diseases. More specifically, owing to the inter-relationship between gut microbiota and bile acid (BA) enterohepatic circulation, the role of changes in this ecosystem that may affect the development and management of liver diseases, such as HCC and cirrhosis, has been investigated (4-6).

In a recent study by Jia et al. (7) the authors have characterized the changes that occurred in 28 patients with intrahepatic CCA (iCCA) and other 28 HCC patients regarding their intestinal microbiota, BA profile and cytokines production in an attempt to identify new markers for the differential diagnosis of iCCA and HCC, which is not always evident in clinical practice. Another interesting aspect investigated in that study was the analysis of vascular invasion (VI), which is an indication of poor prognosis in iCCA patients, whose overall survival is reduced by half when VI occurs.

Using profiling of $16 \mathrm{~S}$ ribosomal RNA gene sequencing and linear discriminant analysis effect size (LEfSe) in their work, Jia et al. have found that the abundance of four genera (Lactobacillus, Actinomyces, Peptostreptococcaceae, and Alloscardovia) was increased in the flora of patients with iCCA compared to those of HCC, cirrhosis and healthy individuals. The combination of the genera Lactobacillus and Alloscardovia allowed them to discriminate between iCCA and the other three conditions investigated. Although these findings, together with the presence of the Alloscardovia genus only in patients with iCCA, are very valuable from a diagnostic point of view, they must be validated in a larger 
cohort of patients. This is the first complete characterization of microbiota composition in iCCA patients, and is in part consistent with the results obtained by Avilés-Jiménez et al. (4), who have reported that in patients with extrahepatic CCA (eCCA) the abundance of Actinomyces in the bile duct is higher than in benign biliary diseases, which could suggest an intestinal origin of the bacteria. The overgrowth of several species of bacteria has already been associated with gastrointestinal cancer, for instance some species belonging to the genera Actinomyces and Peptostreptococcaceae in colorectal cancer, whereas species of other genera, such as Lactobacillus plantarum have been proposed to protect against HCC development.

The relationship between gut microbiota and BA metabolism is well known since intestinal bacteria play a key role in the generation of the wide variety of molecular species present in our BA pool (8). Thus, the human liver synthesizes from cholesterol, mainly cholic acid (CA) and chenodeoxycholic acid (CDCA), which are conjugated with glycine (GCA and GCDCA) or taurine (TCA and TCDCA). During their intestinal transit, these primary BAs undergo mostly three types of transformations that involve enzymes of the intestinal bacteria: (I) deconjugation by bile salt hydrolases (BSHs); (II) $7 \alpha$-dehydroxylation, whereby bacterial dehydratases remove the hydroxyl group at $\mathrm{C} 7$ position to generate secondary BAs, deoxycholic acid (DCA) and lithocholic acid (LCA) from CA and CDCA, respectively; and (III) oxidation/epimerization of the hydroxyl groups at positions C3, C7 and C12, performed by hydroxysteroid dehydrogenases enzymes (HSDHs). The epimerization of $7 \alpha-\mathrm{OH}$ from CDCA to $7 \beta-\mathrm{OH}$ is the origin of ursodeoxycholic acid (UDCA). While deconjugation and oxidation reactions are carried out by a broad spectrum of anaerobic bacteria, C7-dehydroxylation of BAs is restricted to a limited number of anaerobic species, such as those of the genus Clostridium.

Several studies have reported changes in serum levels of some molecular species of BAs in CCA patients, but none of these changes have been proposed as unequivocal indication of the presence of this tumor $(3,9)$. Although variations in the composition of the BA pool are described, there is a controversy regarding whether the total BA pool is increased or decreased in these patients (9-11). The most important changes described were an increase in GCA (9-11), a decrease in TCDCA (9), LCA and DCA (11), together with changes in the ratio of glycoconjugated-to-tauroconjugated species (12) and in the proportion of free BAs compared to conjugates (9). However, in most of these studies the information on the type of CCA (iCCA vs. eCCA) was not provided, which precludes any comparison with the study by Jia et al., in which the authors have analyzed by HPLC-MS/ MS the levels of $16 \mathrm{BA}$ molecular species in both plasma and feces without finding any significant differences in BA profile to discriminate between iCCA and the other groups of individuals. For this reason, they looked for another approach, the determination of the plasma-stool ratio (PSR). They reported that both the PSR of glycoursodeoxycholic acid (GUDCA) and tauroursodeoxycholic acid (TUDCA) were differentially elevated in patients with iCCA compared with those of HCC, cirrhosis, and healthy individuals. The PSR for TUDCA also showed a strong positive correlation with the abundance of Lactobacillus and Alloscardovia.

Although these findings are interesting and allow the authors to propose future studies in which their diagnostic utility could be confirmed by increasing the number of patients analyzed, their interpretation and physiological and clinical significance are complex. The main concern is the use of the unusual parameter PSR. An acceptable ratio between two compartments could be calculated using concentrations or total amounts of BAs in both compartments, or even concentration in one compartment (blood) vs. the total amount in the other (feces). However, in the case of the stool the concentration is not an acceptable parameter because for the same amount of BAs the volume of feces is quite variable among samples of the same individual.

Another controversial point to be considered is the causeeffect relationship of the observed changes. It is unclear whether iCCA induces alterations in BA homeostasis, due for instance to impaired or enhanced expression of liver BA transporters and enzymes involved in BA metabolism, and hence changes intestinal BA pool composition, which causes certain bacterial species to predominate and depressing the growth of others or just the opposite, i.e., iCCA induces dysbiosis, due to changes in environmental conditions (diet, drugs, infections, inflammation), and this is the responsible for BA pool modification. Indeed, intestinal inflammatory diseases have been often associated with dysbiosis and subsequent reduction in the ability to generate secondary BAs, which are potent agonists of nuclear receptors involved in the control of essential genes in BA homeostasis. To close the circle, it should be taken into account that BAs are signaling molecules involved, through specific nuclear (e.g., FXR) and membrane (e.g., TGR5) receptors and growth factors (e.g., FGF19), in the regulation of cell growth and proliferation, which accounts for their role as co-carcinogens 
in the promotion of CCA (13).

Because some BA species are able of inducing inflammatory processes through TGR5 and FXR receptors, and since in the group of iCCA patients with VI an increase in the serum levels of GCDCA, TCA, GDCA, TDCA and TUDCA was observed by Jia et al., these authors decided to determine the levels of cytokines involved in this process. The analysis revealed that iCCA patients with VI had higher levels of IL-4 compared with those without VI. Plasma levels of TNF- $\alpha$, IL-10, TGF- $\beta 1$, TGF- $\beta 2$, IL- $1 \beta$, MCP-1 and histamine were similar in both groups. On the other hand, in this study the levels of IL-6, as well as those of CDCA, were lower in the group with VI than in the nonVI group, which is not fully in agreement with previous observations on the association between BA accumulation, the increase in IL-6 and downregulation of FXR (13) and CYP7A1 $(14,15)$, the key enzyme for BA synthesis.

In conclusion, in the paper by Jia et al. the existence of interrelationships among gut microbiota, BA profile, serum levels of cytokines and iCCA patient outcome has been investigated. Although the study contains valuable results, it seems evident that to reach a consensus on the validity of new markers for the diagnosis, prognosis and progression of the disease it is required to develop combined algorithms, instead of using single parameters, and to expand the study to a greater number of patients.

\section{Acknowledgments}

Funding: Carlos III Institute of Health, Spain (PI16/00598 and PI19/00819) co-financed by European Regional Development Fund; Ministry of Science and Innovation, Spain (SAF2016-75197-R); Asociación Española Contra el Cancer, Spain (AECC-Cancer raros 2017/2020) and Centro Internacional sobre el Envejecimiento, Spain (OLD-HEPAMARKER, 0348_CIE_6_E) co-financed with European Union ERDF funds.

\section{Footnote}

Conflicts of Interest: All authors have completed the ICMJE uniform disclosure form (available at https://hbsn. amegroups.com/article/view/10.21037/hbsn.2019.10.11/coif). The authors have no conflicts of interest to declare.

Ethical Statement: The authors are accountable for all aspects of the work in ensuring that questions related to the accuracy or integrity of any part of the work are appropriately investigated and resolved.

Open Access Statement: This is an Open Access article distributed in accordance with the Creative Commons Attribution-NonCommercial-NoDerivs 4.0 International License (CC BY-NC-ND 4.0), which permits the noncommercial replication and distribution of the article with the strict proviso that no changes or edits are made and the original work is properly cited (including links to both the formal publication through the relevant DOI and the license). See: https://creativecommons.org/licenses/by-nc$\mathrm{nd} / 4.0 /$.

\section{References}

1. Macias RIR, Banales JM, Sangro B, et al. The search for novel diagnostic and prognostic biomarkers in cholangiocarcinoma. Biochim Biophys Acta Mol Basis Dis 2018;1864:1468-77.

2. Marin JJG, Lozano E, Herraez E, et al. Chemoresistance and chemosensitization in cholangiocarcinoma. Biochim Biophys Acta Mol Basis Dis 2018;1864:1444-53.

3. Banales JM, Iñarrairaegui M, Arbelaiz A, et al. Serum metabolites as diagnostic biomarkers for cholangiocarcinoma, hepatocellular carcinoma, and primary sclerosing cholangitis. Hepatology 2019;70:547-62.

4. Avilés-Jiménez F, Guitron A, Segura-López F, et al. Microbiota studies in the bile duct strongly suggest a role for Helicobacter pylori in extrahepatic cholangiocarcinoma. Clin Microbiol Infect 2016;22:178.e11-22.

5. Chng KR, Chan SH, Ng AHQ, et al. Tissue microbiome profiling identifies an enrichment of specific enteric bacteria in opisthorchis viverrini associated cholangiocarcinoma. EBioMedicine 2016;8:195-202.

6. Ponziani FR, Nicoletti A, Gasbarrini A, et al. Diagnostic and therapeutic potential of the gut microbiota in patients with early hepatocellular carcinoma. Ther Adv Med Oncol 2019;11:1758835919848184.

7. Jia X, Lu S, Zeng Z, et al. Characterization of gut microbiota, bile acid metabolism, and cytokines in intrahepatic cholangiocarcinoma. Hepatology 2019. [Epub ahead of print].

8. Marin JJ, Macias RI, Briz O, et al. Bile acids in physiology, pathology and pharmacology. Curr Drug Metab 2015;17:4-29.

9. Song WS, Park HM, Ha JM, et al. Discovery of 
glycocholic acid and taurochenodeoxycholic acid as phenotypic biomarkers in cholangiocarcinoma. Sci Rep 2018;8:11088.

10. Proungvitaya S, Sombattheera S, Boonsiri P, et al. Diagnostic value of serum bile acid composition patterns and serum glycocholic acid levels in cholangiocarcinoma. Oncol Lett 2017;14:4943-8.

11. Wang B, Chen L, Chang HT. Potential diagnostic and prognostic biomarkers for cholangiocarcinoma in serum and bile. Biomark Med 2016;10:613-9.

12. Jusakul A, Khuntikeo N, Haigh WG, et al. Identification of biliary bile acids in patients with benign biliary diseases, hepatocellular carcinoma and cholangiocarcinoma. Asian
Pac J Cancer Prev 2012;13 Suppl:77-82.

13. Lozano E, Sanchez-Vicente L, Monte MJ, et al. Cocarcinogenic effects of intrahepatic bile acid accumulation in cholangiocarcinoma development. Mol Cancer Res 2014;12:91-100.

14. Merrell MD, Nyagode BA, Clarke JD, et al. Selective and cytokine-dependent regulation of hepatic transporters and bile acid homeostasis during infectious colitis in mice. Drug Metab Dispos 2014;42:596-602.

15. Rau M, Stieger B, Monte MJ, et al. Alterations in enterohepatic Fgf15 signaling and changes in bile acid composition depend on localization of murine intestinal inflammation. Inflamm Bowel Dis 2016;22:2382-9.

Cite this article as: Herraez E, Romero MR, Macias RIR, Monte MJ, Marin JJG. Clinical relevance of the relationship between changes in gut microbiota and bile acid metabolism in patients with intrahepatic cholangiocarcinoma. HepatoBiliary Surg Nutr 2020;9(2):211-214. doi: 10.21037/hbsn.2019.10.11 